\title{
Historia de la Revista Electrónica Educare de la Universidad Nacional, Costa Rica
}

\author{
María Elena Camacho Villalobos ${ }^{\mathbf{1}}$ \\ Universidad Nacional de Costa Rica (Costa Rica) \\ maria_e01@yahoo.com
}

Recepción: 23/07/2012

Evaluación: $13 / 10 / 2012$

Aceptación: 03/11/2012

Artículo de Reflexión

DOI: http://dx.doi.org/ 10.9757/Rhela.20.03

\section{RESUMEN}

El objetivo de este trabajo es describir la historia de la Revista Educare y sus aportes al ámbito académico y profesional. Entre sus principales logros están: la mejora en su difusión, su visibilidad, la inclusión de parámetros de calidad internacional y el ingreso a reconocidos catálogos internacionales. La Revista proyecta la formulación de un plan estratégico para

\section{History of the Electronic Magazine Educare of the National University of Costa Rica}

\section{ABSTRACT}

The aim of this work is to describe the history of the magazine Educare and its contributions to the academic and professional environment. Among its major achievements are: the improvement in its dissemination, visibility, the inclusion of international quality parameters and the income to recognized international catalogs. The magazine projects the formulation of a strategic plan to increase visibility, scope, social, professional and academic impact.

Key Words: History of Latin American Education Journal, electronic journal educare, history, visibility, open access, periodic publication, indexing. incrementar visibilidad, alcance e impacto académico, social y profesional.

Palabras clave: Revista Historia de la Educación Latinoamericana, Revista Electrónica Educare, historia, visibilidad, acceso abierto, publicación periódica, indexación.

\section{História da Revista Eletrônica Educare da Universidade Nacional de Costa Rica}

\section{RESUMO}

O objetivo deste trabalho é descrever a história da Revista Educare e suas contribuições no âmbito acadêmico e profissional. Entre seus principais êxitos estão: a melhora em sua difusão, visibilidade, a inclusão de parâmetros de qualidade internacional e o ingresso em reconhecidos catálogos internacionais. A Revista projeta a formulação de um plano estratégico para incrementar visibilidade, alcance e impacto acadêmico, social e profissional.

Palavras-chave: Revista História da Educação Latino-americana, Revista Eletrônica Educare, história, visibilidade, aceso aberto, publicação periódica, indexação.

\footnotetext{
1 Es Máster en Administración Universitaria. Labora en la Universidad Nacional de Costa Rica. Actualmente es la directora de la Revista Electrónica Educare. La Dirección es: Universidad Nacional de Costa Rica. Centro de Investigación y Docencia en Educación, CIDE. Revista Electrónica Educare. Heredia, Costa Rica.
} 


\section{INTRODUCCIÓN}

La Revista académica es un medio de comunicación idóneo para difundir el conocimiento. Según López “(...) este tipo de publicación es un instrumento privilegiado para la comunicación entre investigadores, que se distingue de otros medios en el ámbito científico por características como periodicidad, diversidad de resultados de investigación en sus contenidos y dictaminación $(\ldots)^{\prime \prime}{ }^{2}$

Dado que uno de los ejes vitales en una universidad es la investigación, una revista deviene en un soporte que ayuda a concretar, transmitir y visibilizar el conocimiento que se genera mediante este quehacer académico. Constituye, entonces, una herramienta reconocida universalmente para la publicación del saber.

Compartir los resultados de la investigación resulta una responsabilidad inherente al trabajo científico, es parte de la tarea académica. Hacerlos públicos en una revista trae beneficios tanto a los autores y autoras, como a las instituciones universitarias. Para el primer grupo representa un estímulo que se reconoce en la carrera académica y en el crecimiento profesional; para los centros educativos, optimación de sus estándares de calidad, visibilidad y proyección.

En cuanto a las publicaciones en Costa Rica, Solano y Díaz ${ }^{3}$ indican que la llegada de imprenta al país en 1830 -en un periodo más tardío que al resto de Latinoamérica- fue de mucha influencia para iniciar con las primeras impresiones de libros y periódicos. La impresión de revistas también se inició tardíamente, a finales del siglo XIX. Según Azulea, Sabás y Smith, citados por Solano y Díaz, la primera revista en México que integra artículos científicos se llamó El Iris en 1826. En Costa Rica, no es sino hasta 1883 que se publica la Revista astronómica y meteorológica, una de las primeras revistas científicas. Luego surgen otras publicaciones y, en 1831 nace la primera revista pedagógica llamada La Enseñanza.

2 Maricela López Ornelas, "Diseño y validación de un instrumento para evaluar revistas académicas electrónicas en Internet" (Tesis Maestría en Ciencias Educativas, Universidad Autónoma de Baja California, 2004), 1-45, 22.

3 Flora J. Solano Chaves y Ronald Eduardo Díaz Bolaños, La ciencia en las revistas científicas, culturales, literarias, pedagógicas y religiosas de Costa Rica (1892-1910) (San José, Costa Rica: SINABI, s. f). http://www.sinabi.go.cr/Biblioteca\%20Digital/ ARTICULOS/Solano\%20Flora\%20y\%20Diaz\%20Ronald/La\%20ciencia\%20en\%20las\%20revistas\%20costarricenses.pdf $(9 / 05 / 2012)$. 
En este recuento histórico no se puede pasar por alto El Repertorio Americano (de 1919 a 1958), publicación de índole cultural y social, dirigida por el ilustre escritor e intelectual, Joaquín García Monge. El Repertorio constituye un hito en la historia cultural del país. Aunque sin una orientación ni contenidos específicos de la disciplina educativa, los más connotados académicos del país y del ámbito latinoamericano escribieron en ella, por lo que resulta innegable su influencia positiva para las revistas académicas, que comenzaron a aparecer a partir de la segunda mitad del siglo XX. ${ }^{4}$ Esta se mantuvo vigente por 39 años, desapareció y luego, en 1974, editada por la Universidad Nacional de Costa Rica, resurgió en una segunda época.

(...) En 1974, la comisión ad-hoc que organizaba la Universidad Nacional, gracias a las gestiones de intelectuales y personalidades del mundo académico (...) se llevó adelante la iniciativa de volver a sacar a la luz la revista Repertorio Americano (...). En un gesto que se calificó como propio del espíritu garciamongiano, los herederos de don Joaquin, la familia García Carillo, en la persona de su hijo el Dr. Eugenio García Carillo, otorgaron a la Universidad Nacional los derechos legales de la revista Repertorio Americano y de esta forma, la Universidad y específicamente el IDELA, como instituto de investigación especializado proponente de la Cátedra Joaquín García Monge, (...) adquirieron el mandato de ser depositarios de esta joya documental (...) convertido, ahora, en revista universitaria, pero que mantendría las características de proyección internacional, así como la visión humanística, latinoamericanista y su condición de publicar misceláneas. ${ }^{5}$

Sobre el tema específico de la educación se crearon, en las instituciones educativas, varias revistas. En ellas se publican artículos en las disciplinas de la educación tanto formal como no formal.

- En la Universidad de Costa Rica: en 1977, Educación Revista de la Universidad de Costa Rica; en 2001, Actualidades Investigativas en Educación, y en 2011, Gestión de la Educación.

4 Arnoldo Mora Rodríguez, "Joaquín García Monge y el Repertorio Americano”. Revista Comunicación Vol: 12 No. 1 y 2 (2003): 162-166. http://www.tec-digital.itcr.ac.cr/servicios/ojs/index.php/comunicacion/article/view/1193/1100 (9/05/2012).

5 Marybel Soto Ramírez, "Las revistas académicas del Instituto de Estudios Latinoamericanos", Revista Repertorio Americano. Segunda nueva época. No. 21 (2011): 334. http://www.revistas.una.ac.cr/index.php/repertorio/article/view/4708 (13 de marzo, 2013) 
- En la Universidad Estatal a Distancia: en 2008, Innovaciones Educativas; en 1998, Revista Educación XXI; en 2010, Revista Electrónica Calidad en la Educación Superior y en 2000, Posgrado y sociedad.

- En la Universidad Nacional: en 2001, la Revista Educare, posteriormente, en 2008, cambia su nombre a Revista Electrónica Educare; y en 2002, Ensayos Pedagógicos.

- En el Ministerio de Educación Pública de Costa Rica: en 2009, Revista Conexiones.

- En el Colegio de Licenciados y Profesores en Letras, Filosofía, Ciencias y Artes: en 1991, la Revista Umbral.

\section{Evolución de las revistas}

De acuerdo con CINDOC-CSIC, las revistas surgen a mitad del siglo XVII con el propósito de comunicar resultados, especialmente de datos en las disciplinas física, química y anatomía: "Les journals fueron adoptados como el medio más idóneo para comunicar nuevos resultados a la comunidad científica". ${ }^{6}$

Tradicionalmente, el medio para publicar los resultados de investigación se daba, en especial, en las revistas con formato impreso; pero, con el advenimiento de Internet por 1990, nacen otras formas para publicar y se da la transición al formato digital.

De acuerdo con Babini:

El uso de Internet y de la Web está cambiando la forma de producir investigaciones, de publicar sus resultados en revistas, $y$ de acceder a las mismas en las bibliotecas. El área publicaciones y las bibliotecas se encuentran en un perído de transición en el cual conviven el formato impreso y el formato digital de las revistas. ${ }^{\text {? }}$

6 CINDOC-CSIC, "Revistas científicas electrónicas", AÑO.12. http://www.erevistas.csic.es/especial_revistas/revistas11.htm (12 de junio, 2013)

7 Dominique Babini, “Revistas académicas: Transición al formato digital”, (2008): párr. 4. http://www.erevistas.csic.es/especial_ revistas/revistas4.htm. (23 de junio de 2013) 
Las revistas impresas por lo general conservan este formato, pero paralelamente implementan el digital, según indica Babini en los resultados de entrevistas realizadas en el 2007 a editores y bibliotecas, en un estudio a 12 instituciones de investigación de EUA y Canadá. Indican que "aproximadamente $60 \%$ de las 20000 revistas arbitradas ya están disponibles en formato digital, y por ahora la norma para las revistas impresas es publicar en ambos formatos". 8

Toma fuerza la tendencia de la publicación de revistas completamente electrónicas. Indica Harrassowitz, citado por Aliaga y Rodríguez, que la primera revista electrónica fue New Horizns in Adult Education, en 1987, Online Journal of Current Clinical Trials en 1992.9

Los estudios de Harter y Kim, citados por Aliaga y Suárez "Encontraron que el área académica que había producido más revistas electrónicas era el [sic] educativo, seguido [sic] por la literatura $(\ldots.){ }^{\prime \prime} \cdot{ }^{10}$ La tecnología trae un cambio socio-cultural que influye todos los ámbitos disciplinarios y, por ende, la producción de revistas, generando importantes beneficios.

\section{Acontecimientos actuales e innovaciones en las revistas electrónicas}

Las publicaciones en línea generan ventajas importantes que superan la publicación con impresión en papel. A continuación se enumeran algunas de ellas:

- La creación de cosechadores de información por medio de la iniciativa de archivos abiertos (Open Archive Initiative-OAI): los cosechadores de información son un sistema digital que por medio de metadatos permite la captación sin dificultad de localizar de una red de divulgación a otra. Según SEDIC:

La iniciativa de archivos abiertos (OAI) proporciona la arquitectura y especificaciones técnicas necesarias para que productores y distribuidores de documentos de acceso abierto pongan a disposición de agrega-

8 Babini, "Revistas académicas", párr. 4.

9 Francisco M. Aliaga y Jesús Suárez Rodríguez, “Tendencias actuales en la edición de revistas electrónicas” RELIEVE Vol.: 8 No. 1 (2008): http://www.uv.es/RELIEVE/v8n1/RELIEVEv8n1_0.htm (19 de junio de 2013)

10 M. Aliaga, et al., “Tendencias actuales”, 2008 http://www.uv.es/RELIEVE/v8n1/RELIEVEv8n1_0.htm 
dores de contenidos metadatos sobre los documentos que almacenan con objeto de hacerlos visibles y accesibles a los investigadores. OAI viene a solucionar el problema de la dispersión de los documentos en múltiples depósitos institucionales, temáticos, revistas individuales, portales de revistas electrónicas, etc. Desde el punto de vista del investigador, hace posible la integración de documentos de una misma temática, área geográfica, tipología, etc. en una única herramienta que es capaz de proporcionarle, además, una serie de valores añadidos, como selección, búsqueda e identificación, evaluación, etc. ${ }^{11}$

- Evita las limitaciones de espacio en el papel: las editoriales restringen el espacio en las publicaciones por los altos costos del papel. Señala Aguirre (1999-1998) que se logra

(...) evitar las limitaciones de espacio que suelen imponer las editoriales. "Espacio" es papel y "papel" es dinero. Las revistas acotan el número de los trabajos y la extensión de los mismos [sic] conforme a los presupuestos de que disponen para la edición. La edición digital no necesita, como es obvio, papel alguno y desaparecen los costes derivados (...) que el material sea modificable o ampliable. En la publicación científica es muy importante la posibilidad de establecer distintas fases en los documentos. Determinados documentos se encuentran en proceso de revisión y se distribuyen para obtener opiniones o contrastes antes de su redacción definitiva. La red es un medio perfecto para este tipo de sistemas (...). ${ }^{12}$

- Tiraje reducido de ejemplares: las editoriales de las universidades buscan reducir la cantidad de ejemplares para bajar costos y adicionalmente no todas las revistas tienen una distribución importante, lo que provoca que se queden almacenadas. Indica Aguirre (1999-1998) que:

(...) como 'medio de difusión' permite: (...) solventar el problema de las bajas tiradas. Una revista científico-universitaria, como media, tiene una tirada que oscila entre los 500 y los 1.000 ejemplares. Son muy pocas las que superan estas cifras. La edición electrónica en red no

11 SEDIC, "La iniciativa de ficheros abiertos (Open Archive Initiative - OAI): protocolo OAI-PMH, proveedores de datos, proveedores de servicios". (s. f.): párr. 1 y 2 . http://www.sedic.es/autoformacion/acceso_abierto/4-iniciativa-ficheros-abiertos. html (16 de setiembre de 2013)

12 Joaquín María Aguirre Romero, "Las revistas digitales y la vida académica” No. 6-7 (1997-1998): 8. http://historia.fcs.ucr.ac.cr/ revista/relectronicas/revistas\%20digitales\%20y\%20vida\%20academica.pdf (11 de setiembre, 2013) 
entiende de "tiradas"; simplemente no existe concepto, ligado al mundo del papel. La revista está ahí para cualquiera que quiera consultarla. ${ }^{13}$

- Distribución limitada limitada de las revistas versus revistas en línea: los canales de distribución más conocidos en las editoriales universitarias son el canje y la suscripción, que muchas veces no alcanzan una labor eficiente, pues esta tarea implica mercadeo y contactos. Aguirre indica que:

El proceso de distribución es muchas veces más complicado (...) no es fácil encontrar distribuidoras dispuestas a ocuparse de [revistas cientificas]. (...). La edición digital en línea no requiere distribuidoras; la red es un medio natural y está disponible para todos sin necesidad de intermediarios (...). La distribución no requiere ningún proceso por parte de los editores. Son los lectores los que acceden a la información y no la información la que ha de llegar a los lectores. ${ }^{14}$

- Cumplimiento de la periodicidad a tiempo: uno de los parámetros de calidad más rigurosos que exigen los índices internacionales es el cumplimiento de la periodicidad, con las revistas en línea indica Aguirre "que no existan números atrasados ni devoluciones. La revista puede mantener todo su material en línea a disposición de sus lectores". ${ }^{15}$

- Búsquedas más eficientes y eficaces: las revistas que usan adecuadamente las palabras clave, controladas por un tesauros, aseguran a las personas usuarias una localización más atinada del material digital. Indica Aguirre que “(...) siempre quedaría a los posibles interesados el recurso de las búsquedas temáticas o por palabras clave que le llevarían directamente al artículo de sus pesquisas". ${ }^{16}$

- Multimedia: uno de los beneficios que marcan diferencia con las revistas impresas es la integración de la multimedia. 
(...) Los lectores también ganan porque las posibilidades que ofrece el formato de hipertexto, con elementos multimedia, hace mucho más atractiva la lectura de un artículo. De hecho, existen publicaciones electrónicas donde las referencias bibliográficas son vinculos a resúmenes en Medline de los artículos citados, y permiten también incluir sonidos, videos, archivos de datos y todo tipo de imágenes sin limite de espacio en los artículos. ${ }^{17}$

- Subir a la red los artículos listos: las nuevas tendencias y el formato digital permiten que los artículos que estén arbitrados y corregidos puedan subirse, sin necesidad de que la revista esté completamente terminada; esto beneficia a las personas autoras puesto que no deben esperar el cierre oficial del número para ver su artículo publicado. Tal como señala Travieso (2003) "Permite la publicación casi inmediata de la información, incluso de forma automática, si así se desea. Con esta ventaja se puede salvar el problema de la obsolescencia de la información científica". ${ }^{18}$

- Las revistas electrónicas permiten crear espacios de discusión: la posibilidad de colocar valor agregado a las publicaciones en línea es un factor que enriquece la academia, tal como indica Mayelín:

La capacidad de crear espacios de discusión, así como de contraste de opiniones y datos en el seno de la comunidad de investigadores. La red facilita la réplica y permite la revisión constante de los documentos. El debate es auténtico, puede mantenerse, si se desea, de forma personalizada con el autor por medio del correo electrónico o de forma abierta mediante los grupos de discusión que pueden crearse para una cuestión específica. ${ }^{19}$

En este contexto, el objetivo de este trabajo es describir la historia de la Revista Educare y sus aportes al ámbito académico y profesional. Con este propósito, se obtuvo información mediante la revisión de: actas de reuniones del Consejo Editorial, comprendidas entre 2000 y 2012; las revistas publicadas desde el 2001 hasta el 2012.

\footnotetext{
17 Mayelín Travieso Aguiar, "Las publicaciones electrónicas: Una revolución en el siglo XXI". ACIMED Vol. 11 No. 2 (2003): 8. http://eprints.rclis.org/5101/1/publlicaciones.pdf. (13 de marzo, 2013)

18 Travieso Aguiar, "Las publicaciones electrónicas", 13. http://eprints.rclis.org/5101/1/publlicaciones.pdf.

19 Travieso Aguiar, "Las publicaciones electrónicas, 13. http://eprints.rclis.org/5101/1/publlicaciones.pdf.
} 


\title{
3. Revista Electrónica Educare
}

\section{Adscripción institucional}

La Revista Electrónica Educare está adscrita directamente al Centro de Investigación y Docencia en Educación (CIDE), de la Universidad Nacional. Este es un centro que desarrolla procesos de investigación educativa, docencia universitaria, y formación docente de grado y posgrado para la educación formal, no formal y educación continua. Se compone de cuatro escuelas: Educación Básica, Educación Rural, Educación para el Trabajo y Educología. También forma parte del CIDE, el Instituto de Estudios Interdisciplinario de la Niñez y la Adolescencia (INEINA) y el Centro de Información y Documentación en Educación, Niñez, Adolescencia y Familia (CIDENAF).

En su misión, el CIDE establece la importancia de realimentar el conocimiento para provocar el mejoramiento cualitativo de la educación, con la finalidad de facilitar el desarrollo del ser humano como miembro activo de la sociedad, tal como señala el exrector Olman Segura Bonilla:

\begin{abstract}
(...) es el compromiso con la formación docente de grado y posgrado para la educación formal, no formal y educación continua, y la producción y divulgación sistemática del conocimiento. Aquí se promueve la reflexión crítica y se establece un compromiso con las comunidades para impulsar el mejoramiento cuantitativo y continuo de la educación, el desarrollo integral de la persona y la transformación social, en los ámbitos institucional, nacional y regional. ${ }^{20}$
\end{abstract}

\section{Su razón}

En el cumplimiento de esta misión, para lograr ese impacto en la sociedad, una publicación periódica con el debido respaldo académico es un instrumento fundamental. Según Meléndez, Jiménez, Labadie y Rodríguez una publicación periódica es aquella que "publica artículos científicos o información de actualidad, o ambos, sobre investigación y desarrollo acerca de un campo científico determinado".21 Por eso, desde sus inicios, el centro se ha preocupado por mantener comunicación con la comunidad nacional e

20 Olman Segura Bonilla, "XXV aniversario del CIDE”. Revista Electrónica Educare Vol.: 12 No. 1 (2008): 5. http://www.revistas.una. ac.cr/index.php/EDUCARE/article/view/1405. (11 de junio de 2013)

21 Dolores Meléndez Suárez et al., MEDISAN: órgano de publicación periódica oficial de los profesionales y técnicos de la salud de Santiago de Cuba Vol.: 11 No. 1 (2007): párr. 1 http://www.bvs.sld.cu/revistas/san/vol11_1_07/san02107.html. (12 de junio de 2013) 
internacional, por medio de una revista. Esta es la razón de ser de Educare, editada ininterrumpidamente y publicada por primera vez en julio del 2001. Educare surge, entonces, con el objetivo de divulgar la producción intelectual generada en el CIDE, así como de la de otros colaboradores externos nacionales e internacionales. Su propósito consiste en construir pensamiento educativo en forma interdisciplinaria y su cobertura temática es la educación en todas sus disciplinas.

\section{Sus inicios}

A la revista Educare la antecedió el Órgano de Comunicación CIDE, que salió a la luz en 1986 y la Revista CIDE, cuyo último número apareció en 1993. El exdecano Eddie Alberto Vargas Rodríguez (1992-1998) señaló que “(...) fue imposible editar la revista del CIDE, que necesitaba de presupuesto para su publicación y para contratar un director; la crisis presupuestaria imposibilitó el desarrollo de un proyecto importante y necesario, no solo para el Centro y la Universidad, sino para el país en general.22

Después de ocho años, un grupo de académicos y académicas, apoyados por el Decano Dr. Miguel Gutiérrez Rodríguez (1998-2003) se reunió para perfilar una nueva publicación, con una visión actualizada, que se dirigiera al personal académico y al estudiantado universitario, así como al profesional que ejerce en el sistema educativo nacional e internacional. Nació, así, la Revista Educare, con soporte en papel.

Sus primeros números fueron apoyados económicamente por el proyecto Programa de Cooperación Académica del gobierno de Holanda (2001-2003). El sello editorial de la Editorial de la Universidad Nacional se le otorgó mediante el oficio RE-49-2000, con fecha 21 de noviembre, 2000.

Paralelamente, los números del 2001 al 2008, incluían un suplemento titulado Educare en el aula. Este, según Rubio , “(...) es una propuesta de mediación pedagógica, una guía para que las y los docentes [sic] construyan conocimiento, junto a sus estudiantes, acerca de algún artículo publicado en la revista (...) debe ser contextualizada a la cultura escolar donde será

22 Eddie Alberto Vargas Rodríguez, "El reto de los años noventa: Sus logros, dificultades y sueños en un contexto de crisis presupuestaria para quienes no éramos prioridad en la universidad”, Revista Electrónica Educare Vol.: 12 No. 1 (2008): 159-165. http://www.revistas.una.ac.cr/index.php/EDUCARE/article/view/1426. (13 de marzo de 2013) 
aplicada"23. El primer suplemento se dedicó al tema "Educación para el amor" y contiene reflexiones escritas por el Dr. Gerardo Steele.

\section{Periodicidad}

En cuanto a la periodicidad, en los primeros años no estaba claramente definida y es a partir del 2008 cuando se regula: una publicación semestral y un número extraordinario. El primer número comprendía de enero a junio. El segundo, de junio a diciembre. Los números extraordinarios se programaban para noviembre.

En el 2012, debido a la cantidad de artículos que ingresan (aproximadamente 100 al año) y por recomendación de la evaluación que realizó SciELO (Scientific electronic library online), se cambia la periodicidad a cuatrimestral, “(...) La periodicidad es un indicador del flujo de la producción científica que depende del área específica tratada por la revista. Es también un indicador relacionado con la oportunidad y velocidad de la comunicación" ${ }^{24}$ También recomienda una periodicidad cuatrimestral para las revistas con área temática de humanidades; y que se publiquen como número deseado 24 artículos por año. ${ }^{25}$ Actualmente Educare en su primer número comprende de enero a abril. El segundo, de mayo a agosto y el tercero de setiembre a diciembre.

\section{Canales de distribución y mercadeo}

Los canales de distribución de la Revista Educare, desde el 2001 hasta el 2007, eran los tradicionales (venta, suscripción, canje y donación). Se realizaron esfuerzos para lograr la suscripción de lectores externos al CIDE, suscripción del personal académico y administrativo del CIDE mediante la deducción de planillas y suscripción de estudiantes. Actualmente esta etapa mejora radicalmente con la conversión de la Revista a formato electrónico. Indica Mayelín Travieso Aguiar que "son muchas las ventajas que ofrece una revista electrónica, especialmente las distribuidas por Internet, en comparación con las versiones impresas (...) entre ellas: capacidad de interacción con los lecto-

\footnotetext{
23 Gerardo Steele Zúñiga y Carlos Rubio Torres, "Educación para el amor”, Revista Educare Vol.: 1 No. 1 (2001): 1.

24 SciELO, "Criterios SciELO-Costa Rica" (San José, Costa Rica: SciELO, 2007), párr. 10. http://www.scielo.sa.cr/avaliacao/ avaliacao_es.htm (15 de marzo, 2013).

25 SciELO, "Criterios SciELO-Costa Rica”, párr.11.
} 
res, publicación y distribución inmediata, bajos costos de producción (...)" ${ }^{26}$ El ingreso de Educare a índices internacionales, la difunde universalmente, tal como indica Saray Córdoba "la presencia de las revistas en esos índices provoca varias consecuencias (...) adquieren mayor visibilidad mundial, facilita el acceso a la Revista desde diversos sitios alrededor del mundo, (...) se logra un mayor intercambio y con ello, se alcanza un mayor desarrollo del campo científico nacional. ${ }^{27}$

En relación con la estrategia de mercadeo, se realizaron presentaciones de la Revista en diferentes partes del país; en estas visitas se presentan conferencias de diversas temáticas: Educación para el amor, Diversidad: retos para la educación, El trabajo en equipo: una alternativa para el cambio en la cultura institucional, entre otras. En este momento, la distribución y difusión se da universalmente, debido al formato electrónico y a la indexación de esta en diversos catálogos internacionales que la visibilizan.

\section{Gestiones de dirección}

El primer director de Educare fue el Dr. Carlos H. Lépiz Jiménez, académico del CIDE, quien dedicó su gestión a definir y consolidar las políticas de este medio, los trámites para su creación y a conformar el primer número. Renuncia a la Dirección en el 2000, para asumir el puesto de Vicerrector Académico.

El segundo director de Educare fue el Lic. Carlos Rubio Torres (2001-2003), a quien le correspondió la publicación del primer número de la Revista Educare. Lo acompañó, en el Consejo Editorial la M. Ba. Alexa Jengich Buch, M. Sc. Miguel Rodríguez, Licda. Xenia Pacheco y el M. Sc. Enrique Zúñiga. En el Consejo Asesor Externo participaban la Licda. María Eugenia Dengo Vargas, Vicky Ramos Quesada, Dr. Alejandro Acuña Limón, Dr. Dominique Hoozemans y Dr. Gilberto Garro. Como asistente administrativo estuvo el señor Julio César Barquero Barquero.

En la dirección del señor Rubio se define el logo de la Revista, diseñado por la artista Vicky Ramos Quesada. Este logo es en espiral, las letras de la

\footnotetext{
26 Travieso Aguiar, "Las publicaciones electrónicas", párr. 1-2.

27 Saray Córdoba González, "Qué; es una revista indexada", Girasol Digital, Vol.: 8 No. 26 (2005): párr. 10-11. www.vinv.ucr.ac.cr/ girasol-ediciones/archivo/girasol26/indexada.htm ((12 de marzo de 2013).
} 
palabra Educare en formato vertical y en tipografía Dellarobia. También se acuerda usar imágenes en la portada y en cada sección. ${ }^{28}$

En esta etapa se realiza un concurso promovido entre los académicos y las académicas del CIDE para darle nombre a la Revista. Se presentaron varios títulos como: Paideia, Convergencia, Punto de encuentro, Diálogo, Debate, Brecha, Búsqueda, Revista de educación del pueblo, Nuevas tendencias, Perspectivas educativas, Proyección educativa, Magisterio, Cátedra, Akademia, Universitas, Acción educativa, reflexiones educativas y Pueblo y educación, Aula, Comunicación y pedagogía, Acción, Maestra, INCIDE, Perspectivas en educación, Enfoques educativos y Enlaces educativos. ${ }^{29} \mathrm{Se}$ seleccionó el nombre Educare, propuesto por la M. Sc. Paulette Baberousse, docente del CIDE. El título escogido corresponde a un verbo latino 'educare', que hace alusión al sentido de alimentar, cuidad, nutrir, instruir, formar y criar. ${ }^{30}$

Se establecieron tres secciones para cada número:

a). En la investigación y la reflexión: incluye artículos derivados de proyectos de investigación.

b). Nuevas propuestas en la práctica pedagógica: incluye artículos derivados de propuestas ejecutadas en la educación preescolar, primaria, secundaria o especial.

c). En búsqueda de nuevos rumbos: incluye recomendaciones de libros relacionados con educación y pedagogía.

En cada número se presenta obra plástica costarricense, que ilustra la portada y algunas páginas internas.

Otros directores que asumieron la dirección de la Revista Educare fueron el Dr. Gilberto Garro Garita, en el periodo 2003-2004. En esta gestión se promueven visitas a las diferentes unidades académicas, con dos propósitos: motivar al personal docente para que se suscriban a la Revista e invitarlos a escribir artículos. Se trabaja por un plan de mercadeo para financiar la Revista, ya que en el 2003 concluye el apoyo financiero que brindada el

28 Consejo Editorial. Revista Educare Reunión N 16 celebrada el 17 de noviembre (1999).

29 Comité Editorial Revista CIDE, Minuta, Sesión N³ celebrada el 24 de marzo de 1999, a las catorce y veinticincol4:25 horas.

30 Revista Educare, “Página legal”, Revista Educare Vol.: 1 No. 17 (2001). 
proyecto MHO de Holanda. ${ }^{31}$ Se realiza una 'encerrona', con el equipo de la Revista y la señora decana Irma Zúñiga, con el fin de elaborar un Plan Estratégico para los próximos años.

El Lic. Rubio regresa a la dirección de la Revista por un corto plazo (2004) y continúa implementado los lineamientos que se habían proyectado anteriormente.

La M.L. Nuria I. Méndez Garita, asume la dirección en el período comprendido entre el 2005 al 2008. En su gestión, trabaja por la difusión regional de este órgano de difusión, producto del cual se publican tres números extraordinarios: Volumen Extraordinario I, 2007, con trabajos de investigación elaborados por personas egresadas de la Maestría en Pedagogía con énfasis en Diversidad en los Procesos Educativos, pertenecientes a la División de Educación Básica. Volumen extraordinario II, 2007, fruto del III Seminario Latinoamericano de Educación Rural: Encuentro de experiencias de indole centroamericana, con trabajos de participantes centroamericanos y latinoamericanos. El Volumen XII, 2008, producto de la Maestría en Educación Rural Centroamericana (MERC), en el proyecto Fortalecimiento de las escuelas rurales de Costa Rica y Centroamérica, contiene reflexiones sobre la educación en zonas rurales de la región, y la conceptualización de la pedagogía rural; estos trabajos son de académicos y académicas centroamericanas. El tiraje de 300 ejemplares es distribuido en Centroamérica.

En su dirección se publican dos números ordinarios (Volumen XII, 1, 2008 y volumen XII, 2, 2008), el número 1 dedicado a la celebración del XXV Aniversario de la creación del Centro de Investigación y docencia en Educación de la Universidad Nacional (CIDE). Se establecen canjes con revistas de universidades como Antofagasta de Chile, Universidad Nacional de Rosario (Argentina), y Universidad Nacional de México. Se promueven lazos con instituciones como Caja de Ande, Vida Plena, entre otras. La directora da talleres de redacción de artículos en el espacio llamado ‘Miércoles Académicos' dedicado por el CIDE para estas actividades.

31 Acta de la sesión ordinaria número 01, celebrada por el Consejo Editorial de la Revista Educare del Centro de Investigación y Docencia en Educción, celebrada el día 6 de marzo del año 2003, a las 15:00 horas. 
Con esta dirección se consolida el primer sitio en línea para la Revista, en él se suben las tablas de contenido, títulos y resúmenes de cada artículo. Se automatiza, por medio de una base de datos, el cobro por suscripción de la Revista. Se regulan las ediciones con el formato APA y la periodicidad (semestral) y se inicia la postulación de la Revista a Latindex, con el fin de que se señalen los aspectos que deben mejorarse para ingresar a este sistema de información. Se cuidan las ediciones con ilustraciones de artistas nacionales, y se da espacio al estudiantado de la Escuela de Arte y Comunicación Visual de la Universidad Nacional.

El M.L. Marco Vargas Montero asumió temporalmente la dirección de la Revista durante los meses de marzo a octubre de 2008. Da seguimiento al proyecto de la administración anterior. Realiza la traducción del Manual APA y elabora un manual con aspectos importantes para la citación y referenciación, deja listo para impresión el volumen XII, 1, 2008.

La MAU. María Elena Camacho Villalobos, asume la dirección a partir del 2008 y hasta el momento está a cargo de la dirección de la Revista Electrónica Educare.

\section{Educare hoy}

\section{El diagnóstico}

En el 2008, al cumplir 7 años de publicación ininterrumpida, las autoridades del CIDE proponen una evaluación para la Revista Educare. Es así como se emprende un extenso trabajo que incluyó un diagnóstico sobre la Revista. En este se determinaron algunos procesos por mejorar y se diseñó una estrategia para remozar esta publicación. Dentro de esta estrategia, se determinaron cuatro puntos básicos: Convertirla en formato electrónico; Optimizar la calidad en forma y contenido; Visibilizarla, Lograr su indización en bases de datos y catálogos internacionales, y así conseguir una distribución mundial; y Apoyar el ambiente, al no publicar más en formato impreso: no más papel.

\section{Conversión a formato electrónico}

A partir del 2008 se transforma la Revista a formato electrónico y se desiste de su publicación en papel. Esta decisión la favorece tanto en cumplimiento de la periodicidad como en la reducción de costos. Según Barrueco Cruz: 
La publicación electrónica debe ser más barata que la impresa, dado que el único coste que se aborda es el de la llamada primera copia. El coste del resto es prácticamente cero, al igual que los costes de distribución. Una vez hecha la inversión inicial en equipamiento informático para instalar un servidor, su mantenimiento es bastante reducido (...). Vijay Jog (Jog, 1995) citado en Barrueco, en un detallado estudio de los costes de distribución de ambos tipos de documentos estimaba que los costes de producir y distribuir una revista electrónica pueden ser de un 28 a un $48 \%$ menores que su equivalente en papel. ${ }^{32}$

En 2011, la Revista Educare migró hacia el sistema Open Journal System (OJS), ya que se tomó en cuenta la versatilidad de este programa y, además, algunos índices como Redalyc, al que la revista pertenece, trabajan con este sistema.

Open Journals Systems (OJS) es un sistema de gestión y edición de revistas electrónicas desarrollado por el Public Knowledge Projet $(P K P)$, un organismo canadiense conformado por las Universidades British Columbia y Simon Frase.

Se trata de software libre (no tiene, por lo tanto, ningún coste) (...) permite controlar todo el proceso de publicación de una revista en formato electrónico, desde el envío de los manuscritos por parte de los autores hasta la publicación final del número. ${ }^{33}$

En síntesis, el formato electrónico favorece a la Revista por cuanto la visibiliza internacionalmente. Asimismo, la adopción del OJS la beneficia, puesto que este programa es un gestor de los procesos de edición y, paralelamente, es una base de datos.

\section{Optimizar la calidad en forma y contenido}

Para mejorar la calidad de esta publicación, se apuntó en dos sentidos: uno, mejorar todos los aspectos de forma; y otro, mejorar los contenidos de los artículos que se publican.

Pero, son las instituciones promotoras de la publicación de estas revistas (universidad, en nuestro caso), las que deben preocuparse para que estas no desaparezcan, sino que, más bien, se fortalezcan. Por esto, en el caso de

\footnotetext{
32 José Manuel Barrueco Cruz, Revistas electrónicas: normalización y perspectivas. Badajoz: Universidad de Valencia, 2000. http:// www.uv.es/ barrueco/badajoz.pdf (15 de marzo de 2013)

33 Sergio Ruiz Pérez y Juan Pablo Alperin, “OJS: Sistema de gestión de revistas electrónicas”, RevistaeSalud.com Vol.: 3 No. 9 (2007): 1. http://www.revistaesalud.com/index.php/revistaesalud/article/view/143/386 (11 de marzo, 2013).
} 
Educare (y de todas las revistas de la Universidad Nacional), la Dirección de Extensión y El Consejo Nacional de Rectores se propuso impulsar el mejoramiento y la publicación de las revistas de la institución, con parámetros de calidad). ${ }^{34}$ Es importante agregar que, a corto plazo, se inaugurará el primer repositorio de la Universidad Nacional, el cual colaborará también en la difusión de estas publicaciones y que define como "repositorio institucional a aquellos servicios prestados por las universidades, al conjunto de la comunidad, para recopilar, administrar, difundir y preservar la producción documental digital generada en la institución, cualquiera que sea su tipología, a través de la creación de una colección digital organizada, abierta e interoperable (...), para garantizar un aumento de la visibilidad e impacto de esta" ${ }^{35}$

Respecto a optimizar la calidad en forma se trabajó en:

- Se incorpora un nuevo diseño.

- Se vigila que la diagramación reúna aspectos estéticos, que se aprovechen los recursos que permite Internet y que incluya los parámetros de forma que exigen los índices internacionales.

- Se incorporan los procesos de revisión filológica.

- Se aplican los procesos de revisión en las traducciones.

- Se revisan las citas, fuentes y referencias.

- Se establece el control de calidad en cada una de las etapas de edición de cada artículo.

En relación con el contenido, se realizó una revisión de los criterios de calidad que evalúan los índices, los cuales toman en cuenta características básicas (mención del cuerpo editorial, antigüedad, tipos de artículos, entre otros); características de presentación de la revista (página de presentación, mención de la periodicidad, tabla de contenido, membrete bibliográfico, entre otros); criterios de gestión y política editorial (sistema arbitraje, evaluadores(as) externos(as), autores(as) externos(as), entre otros); características de los contenidos (originalidad, instrucciones a los autores, elaboración de referencias bibliográficas, entre otros).

34 Dirección de Extensión y CONARE, "Programa de mejoramiento de la calidad de las publicaciones académicas con énfasis en revistas de universidades públicas costarricenses”. Heredia, Costa Rica: Universidad Nacional.

35 José Manuel Barrueco y Cristina Garcí Testal, "Repositorios institucionales universitarios: evolución y perspectivas”, Fesabid Vol.: 1, No. 11 (2009): 100. http://www.fesabid.org/zaragoza2009/actas-fesabid-2009/99-107.pdf (12 de marzo, 2013). 
En concreto, en cuanto al contenido se han realizado ajustes en:

- Los criterios de calidad establecidos por índices internacionales.

- Cuerpo de arbitrajes externos preferiblemente internacionales.

- Autores(as) externos(as).

- Revisión por pares con la modalidad de doble ciego.

- Evitar la endogamia.

- Detectar el plagio y autoplagio.

- Revisión estricta de citas y referencias bibliográficas.

- Digitalización de todas las revistas que se publicaron con soporte en papel.

- Integración de la carta en que conste que el artículo es inédito, original y la cesión de derechos de autor.

- Sometimiento de la Revista a evaluaciones periódicas.

- Indexación de la Revista en todos los índices posibles.

- Adicionalmente, protección de la Revista y cada artículo con la licencia Creative Commons, adaptada para Costa Rica.

Creative Commons es una organización no gubernamental sin fines de lucro que desarrolla planes para ayudar a reducir las barreras legales de la creatividad, por medio de nueva legislación y nuevas tecnologías (...) buscan restablecer un equilibrio entre los derechos de los autores, las industrias culturales y el acceso del público a la cultura pormedio de la cesión expresa de cierto derechos sobre las obras (...).36

\section{Visibilidad}

Según Buela-Casal ${ }^{37}$ expone que para incrementar el impacto de las revistas y sus publicaciones se debe velar en orden de prioridad por los siguientes aspectos: "1. Incrementar la difusión de la revista, incluirla la revista en el mayor número posible de bases de datos, 3. publicar artículos polémicos, 4. publicar revisiones, 5. publicar en idioma inglés, 6. publicar artículos sobre temas de actualidad, 7. publicar artículos de autores muy citados, 8. establecer acuerdos con medios de comunicación, 9. recomendar que se

36 Latindex UCR. Repositorio de las revistas científicas de la Universidad de Costa Rica, Creative Commons (San José, Costa Rica: Universidad de Costa Rica, s. f.). párr. 1. http://www.latindex.ucr.ac.cr/creativecommons.php (15 de marzo, 2013).

37 Gualberto Buela-Casal, "Evaluación de la calidad de los artículos y de las revistas científicas: Propuesta del factor de impacto ponderado y de un índice de calidad". Psicotherma Vol.: 15 No. 1 (2003): 24. http://www.psicothema.com/pdf/400.pdf (25 noviembre, 2013). 
citen trabajos publicados en la misma Revista, 10. facilitar el acceso a los artículos por Internet.

Para mejorar la visibilidad de la Revista Educare se tomaron las siguientes acciones concretas como:

- Su postulación para evaluación en diferentes índices. Producto de esta valoración fue incluida en: SciELO, Redalyc, Latindex, CLASE, DIALNET, E-REVIST@S, DOAJ, IRESIE, OAI, OEI, Journal TOCs, Sherpa/Romeo, DRJI, y Actualidad Iberoamericana.

- La transformación en formato electrónico.

- Inclusión en el portal de revistas académicas de la Universidad Nacional.

- Inscripción en buscadores como: Google Scholar (buscador especializado en artículos de revistas científicas).

- Inscripción en Google Académico (buscador de bibliografía especializada).

- Inclusión en la red social "Facebook".

- Realización de su gestión editorial desde el Open Journal Systems (OJS).

\section{Impacto social}

Sobre el impacto de la Revista Educare, se rescatan algunos aspectos importantes, como por ejemplo: una difusión internacional; una revista sin costos para el lector, ya que es totalmente gratuita (acceso abierto); un impacto ecológico al no imprimirse; un formato interactivo, con movimiento, color, sonido, además de tener algunos valores agregados como buscadores y otros aditivos. Llega a todo público. Todas estas ventajas logradas se reafirman con lo señalado por Roberto Reyna, citado por Cordero, Organista, López y Nishikawa, quien indica como ventajas las siguientes: "reducción de costos, alcance internacional, disponibilidad inmediata, ligas a otros documentos, retroalimentación de los usuarios, rapidez de publicación. ${ }^{38}$

38 Graciela Cordero Arroyo, Javier Organista Sandoval, Maricela López Ornelas y Kiyoko Nishikawa Aceves, "Revista impresa o electrónica. ¿Cuál es la alternativa? El caso de la revista de investigación educativa (REDIE) No. 25 (2005): 35. http://eprints.rclis. org/15813/1/Organista-et\%20al-2005.pdf (12 de marzo de 2013). 


\section{CONCLUSIÓN}

La permanencia de la Revista Educare en el quehacer educativo, desde sus inicios, resulta innegable. En estos momentos, después de una carrera construida en el camino de variedad de actores y actoras, se recogen frutos en los siguientes logros y ventajas de la pertenencia a prestigiosos índices y su publicación en formato electrónico.

Mayor visibilidad e impacto. La Revista ha mejorado las descargas de consulta, por países especialmente de Latinoamérica, entre ellos: México, Colombia, Venezuela, Perú, Ecuador, República Dominicana, Argentina y de Europa, específicamente, España, y del territorio nacional (42,22 \%). Esta mayor visibilidad de autores y autoras, tanto en su país de origen como en el exterior favorece el sistema de comunicación.

Fácil acceso a las publicaciones. Este aspecto se facilitó por medio del gestor de edición con el que se administra la Revista (OJS), también por ser una publicación de acceso abierto (Open Access), es decir, que el conocimiento se difunde de manera libre y gratuita; la indización y los buscadores que ofrece el sitio de la Revista, facilitan la localización de artículos ágilmente.

Aumento en la rapidez de distribución de artículos. La Revista, al cumplir con el criterio de calidad 'cumplimiento de la periodicidad', provoca que esta se suba al sitio en las fechas correspondientes, así se puede consultar de manera inmediata la información.

Preservación de la información a largo plazo. El formato electrónico permite la preservación de los artículos, pues la Universidad opera con respaldos seguros. Adicionalmente, para preservar las revistas que se imprimieron en papel, se digitalizaron.

Fácil navegación. El acceso a la Revista es de operación sencilla en la plataforma OJS,y se procura que su camino de migas no supere más de tres clic para buscar información.

Interacción más dinámica. El sitio de la Revista y los artículos que la conforman sesuben con interacción de la multimedia, los cuales tienen sonido, enlaces de hipertexto, color. 
Acceso a estadísticas. Educare está inscrita en Google Analytics y Google Scholar, que son buscadores que arrojan estadísticas muy detalladas, por ejemplo: los más citados, mas consultados (cada hora, por día, semana y mes); cuáles países y sus ciudades consultan la Revista, cuánto tiempo se mantienen en la consulta; la tecnología que utilizan los usuarios y usuarias (computadora, celular, etc.).

Contribución con el ambiente y la ecología del planeta. Su conversión a formato electrónico eliminó el uso de papel.

Posibilidad de ser consultada desde cualquier espacio. La Revista puede ser consultada desde cualquier computadora, teléfono móvil y tabletas.

Buscadores muy eficientes. Esta Revista se puede localizar por número, autor, título. Hipervínculos en las referencias o enlaces activos, que permiten localizar los documentos de manera inmediata tan solo con hacer clic sobre ellos. Este tipo de búsqueda supera los sistemas tradicionales de localización en una biblioteca convencional.

Con el propósito de servicio oportuno y eficiente para la comunidad científica que colabora con sus artículos y para la población usuaria, la Revista Electrónica Educare continúa en ese camino de abrir espacios para la comunicación del saber, con la mirada puesta en un horizonte donde este conocimiento ayude a construir un mundo mejorar.

\section{FUENTES}

Acta de la sesión ordinaria número 01, celebrada por el Consejo Editorial de la Revista Educare del Centro de Investigación y Docencia en Educción, celebrada el día 6 de marzo del año dos mil tres, a las quince horas (2003).

Comité Editorial Revista CIDE, Minuta, Sesión №3, celebrada el 24 de marzo de 1999, a las 14:25 horas.

Consejo Editorial. Revista Educare. Reunión Nº 16 celebrada el 17 de noviembre (1999).

\section{REFERENCIAS}

Babini, Dominique. "Revistas académicas: Transición al formato digital", Revistas Científicas Electrónicas (2008). http:/ / www.erevistas.csic.es/especial_revistas/revistas4. htm. (23 de junio de 2013) 
Barrueco Cruz, José Manuel. Revistas electrónicas: normalización y perspectivas, (2000): 14-15. http: / / www.uv.es /\%7Ebarrueco/badajoz.pdf (15 de marzo, 2013).

Barrueco Cruz, José Manuel y Cristina García Testal, "Repositorios institucionales universitarios: evolución y perspectivas", Jornadas Españolas de Documentación, Fesabid Vol.: 1, No. 11 (2009): 99-108. http:/ / dialnet.unirioja.es / servlet/articulo?codigo=2972780 (8 de marzo de 2013).

CINDOC-CSIC, "Revistas científicas electrónicas", (s. f.), párr. 12 http: / / www.erevistas. csic.es/especial_revistas/revistas11.htm. (12 de junio, 2013)

Buela-Casal, Gualberto. “Evaluación de la calidad de los artículos y de las revistas científicas: Propuesta del factor de impacto ponderado y de un índice de calidad". Psicotherma Vol.: 15 No. 1 (2003): 23-35. http: / / www.psicothema.com/pdf/400.pdf

Córdoba González, Saray. "Qué; es una revista indexada", Girasol Digital, Vol.: 8 No. 26 (2005) www.vinv.ucr.ac.cr/girasol-ediciones/archivo/girasol26/indexada.htm $\quad(12$ de marzo de 2013).

Córdoba González, Saray y Rolando Coto. Sobre el Sistema OJS ("Open Journal System"). (San José, Costa Rica: Universidad de Costa Rica, 2008): 1-11. http://www.latindex.ucr.ac.cr/ docs/propuestaOJS.pdf

Cordero Arroyo, Graciela, Javier Organista Sandoval, Maricela López Ornelas, y Kiyoko Nishikawa Aceves. "Revista impresa o electrónica. ¿Cuál es la alternativa? El caso de la revista de investigación educativa (REDIE). Pixel BIT". Revista de Medios y Educación No. 25 (2005): 33-43. http: / / www.redalyc.org/ articulo.oa?id=36802503 (12 de marzo de 2013).

Dirección de Extensión y CONARE, "Programa de mejoramiento de la calidad de las publicaciones académicas con énfasis en revistas de universidades públicas costarricenses". Heredia, Costa Rica: Universidad Nacional.

Fournier Ugarte, Óscar, y Meilyn Garro. ¿Revista digital o impresa? (San José, Costa Rica: Universidad de Costa Rica, s. f.): 1-34. http:/ / www.latindex.ucr.ac.cr/ docs/OJS.pdf

M. Aliaga Francisco y Jesús Suárez Rodríguez. “Tendencias actuales en la edición de revistas electrónicas" RELIEVE Vol.: 8 No. 1 (2002): 3-11. Aguirre Romero Joaquín María. "Las revistas digitales y la vida académica" Cuadernos de documentación multimedia No. 6-7 (19971998): 6-7http: / / historia.fcs.ucr.ac.cr / revista / relectronicas / revistas\%20digitales $\% 20 \mathrm{y} \% 20$ vida\% 20academica.pdf (11 de setiembre, 2013).

Latindex UCR. "Repositorio de las revistas científicas de la Universidad de Costa Rica". Creative Commons (San José, Costa Rica: Universidad de Costa Rica, s. f.). http:/ /www. latindex.ucr.ac.cr/creativecommons.php (15 de marzo, 2013).

López Ornelas, Maricela. "Diseño y validación de un instrumento para evaluar revistas académicas Electrónicas en Internet". (Tesis Maestría en Ciencias Educativas, Universidad Autónoma de Baja California, 2004).

Meléndez Suárez, María Elena, Dolores Jiménez Arias, Sandra Labadié Castillo, y Rafael Rodríguez Castillo. MEDISAN: órgano de publicación periódica oficial de los profesionales y técnicos de la salud de Santiago de Cuba Vol. 11 No. 1 (2007): s. p.

Mayelín Travieso Aguiar, "Las publicaciones electrónicas: Una revolución en el siglo XXI". ACIMED Vol. 11 No. 2 (2003): 1-28. http:/ / eprints.rclis.org/5101/1/publlicaciones.pdf

Mora Rodríguez, Arnoldo. "Joaquín García Monge y el Repertorio Americano". Revista Comunicación Vol.: 12 No. 1 y 2 (2003): 162-166. 
http: / / www.tec-digital.itcr.ac.cr/ servicios / ojs / index.php/comunicacion/article/view / $1193 / 1100$ (9 de mayo, 2013)

Revista Educare. "Página legal”. Revista Educare Vol.: 1 No. 17 (2001).

Ruiz Pérez, Sergio y Juan Pablo Alperin. “OJS: Sistema de gestión de revistas electrónicas”. RevistaeSalud.com Vol.: 3 No. 9 (2007): 1.-12 http:/ / www.revistaesalud.com/index.php/ revistaesalud/article/view/143/386 (11 de marzo, 2013)

SciELO, Criterios SciELO-Costa Rica. San José, Costa Rica: SciELO, 2007. http://www. scielo.sa.cr/avaliacao/avaliacao_es.htm (15 de marzo, 2013).

SEDIC, La iniciativa de ficheros abiertos (Open Archive Initiative - OAI): protocolo OAI$\mathrm{PMH}$, proveedores de datos, proveedores de servicios. (s. f.): párr. 1 y 2. http: / / www.sedic. es/autoformacion/acceso_abierto/4-iniciativa-ficheros-abiertos.html (16 de setiembre de 2013).

Segura Bonilla, Olman. "XXV aniversario del CIDE". Revista Electrónica Educare Vol.: 12 No. 1 (2008): 5-7. http://www.revistas.una.ac.cr/index.php/EDUCARE/article/view/1405 (11 de junio de 2013)

Solano Chaves, Flora J. y Ronald Eduardo Díaz Bolaños. La ciencia en las revistas científicas, culturales, literarias, pedagógicas y religiosas de Costa Rica (1892-1910). San José, Costa Rica: SINABI, s. f. http://www.sinabi.go.cr/Biblioteca\%20Digital/ARTICULOS/Solano $\% 20$ Flora $\% 20 \mathrm{y} \%$ 20Diaz $\%$ 20Ronald / La\%20ciencia\%20en $\%$ 20las\%20revistas $\% 20$ costarricenses. pdf (9 de mayo, 2013)

Soto Ramírez, Marybel. “Las revistas académicas del InstitutodeEstudios Latinoamericanos”. Revista Repertorio Americano. Segunda nueva época. No. 21 (2011): 333-338. http://www. revistas.una.ac.cr/index.php/repertorio/article/view/ 4708 (13 de marzo, 2013).

Steele Zúñiga, Gerardo y Carlos Rubio Torres. "Educación para el amor". Revista Educare Vol.: 1 No. 2 (2001): 1-24.

Travieso Aguiar, Mayelín. "Las publicaciones electrónicas: una revolución en el siglo XXI". ACIMED Vol.: 11 No. 2 (2003): Revista electrónicas, párr. 1-2. http:/ / scielo.sld.cu/scielo. php?script=sci_arttext\&pid=S1024-94352003000200001\&lng=es\&nrm=iso\&tlng=es $(13$ de marzo, 2013)

Vargas Rodríguez, Eddie Alberto. "El reto de los años noventa: Sus logros, dificultades y sueños en un contexto de crisis presupuestaria para quienes no éramos prioridad en la universidad". Revista Electrónica Educare Vol.: 12 No. 1 (2008): 159-165. http:/ /www. revistas.una.ac.cr/index.php/EDUCARE/article/view/1426 (13 de marzo de 2013)

Camacho Villalobos, María Elena. "Historia de la revista electrónica educare de la Universidad Nacional, Costa Rica". Revista Historia de la Educación Latinoamericana. Vol. 15 No, 20, (2013): 71 - 94. 

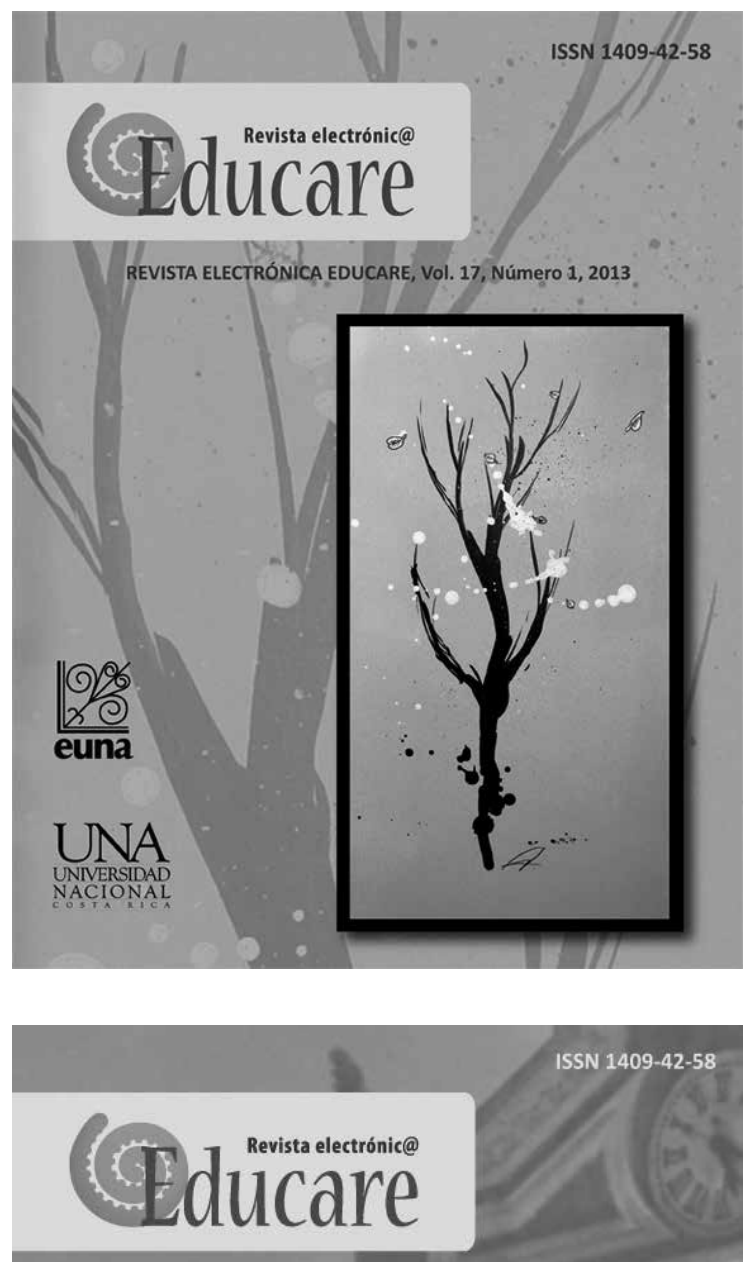

ISSN 1409-42-58

REVISTA ELECTRÓNICA EDUCARE Vol. 17, Número 2, 2013

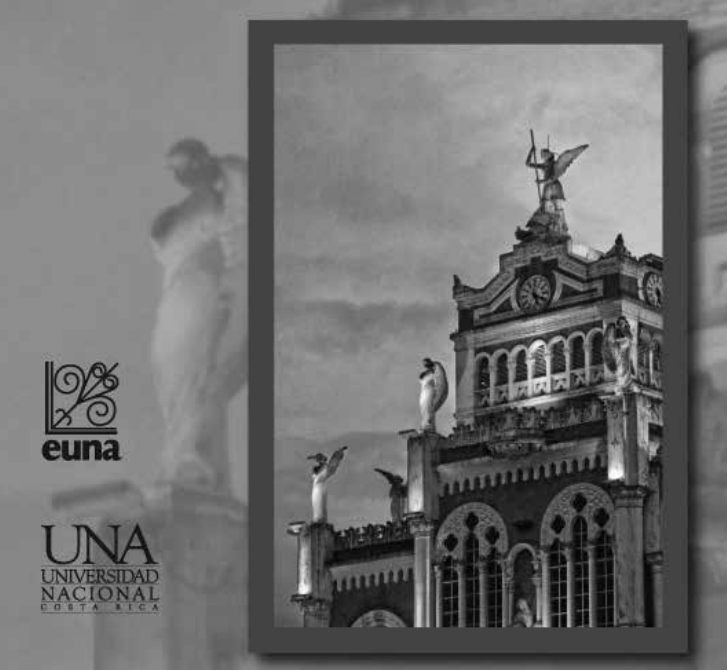

